\title{
Intratumoral Heterogeneity in Uveal Melanoma
}

\author{
Cristina Fonseca $^{a}{ }^{a}$ Rita Pinto-Proença ${ }^{b}$ Sabrina Bergeron ${ }^{b}$ Luís Miguel Pires $^{c}$ \\ Júlia Fernandes $^{\mathrm{a}}$ Isabel Marques Carreira ${ }^{\mathrm{c}-\mathrm{e}}$ Miguel N. Burnier ${ }^{\mathrm{b}}$ Rui Proença ${ }^{\mathrm{a}}$ \\ a Ocular Oncology Reference Center, Centro Hospitalar Universitário de Coimbra, Coimbra, Portugal; \\ ${ }^{b}$ MUHC - McGill University Ocular Pathology and Translational Research Laboratory, Montreal, QC, Canada; \\ 'Genomics and Cytogenetics Laboratory, Faculty of Medicine, University of Coimbra, Coimbra, Portugal; \\ ${ }^{\mathrm{d}} \mathrm{CIBB}$ - Center for Innovative Biomedicine and Biotechnology, University of Coimbra, Coimbra, Portugal; \\ ${ }^{\mathrm{e}} \mathrm{iCBR}-\mathrm{CIMAGO}$ - Center of Investigation on Environment Genetics and Oncobiology, Faculty of Medicine, \\ University of Coimbra, Coimbra, Portugal
}

\section{Established Facts}

- Tumor biopsies in uveal melanoma (UM) are important for estimating prognosis.

- Intratumoral heterogeneity and sample representativity are major concerns.

\section{Novel Insights}

- UM patients treated with conservative methods showed heterogeneity of the clinical response observed on morphologically different areas of the tumor.

- Enucleated specimen showed different pathological patterns and genetic aberrations in morphological distinct areas of the tumor.

\section{Keywords}

Uveal melanoma $\cdot$ Tumor heterogeneity $\cdot$ Prognosis · Biopsy

\section{Abstract}

Tumor biopsies in uveal melanoma (UM) serve mainly the purpose of prognostication and assessment of individual metastatic risk, but can be used for diagnosis in selected cases. The importance of precise information is paramount for selecting adequate surveillance protocols, patient counsel- ing, and optimization of treatment strategies. However, intratumoral heterogeneity and sample representativity are major concerns and can interfere with the correct prediction of the patient's prognosis. We report a series of cases of UM with distinct morphologically identifiable areas, highlighting the differences in clinical behavior, as well as histopathological and genetic features.

(c) 2020 S. Karger AG, Basel

\section{J.F. deceased in November 2019.}


Table 1. Summary of demographic, clinical, pathologic, and genetic data of the 3 cases

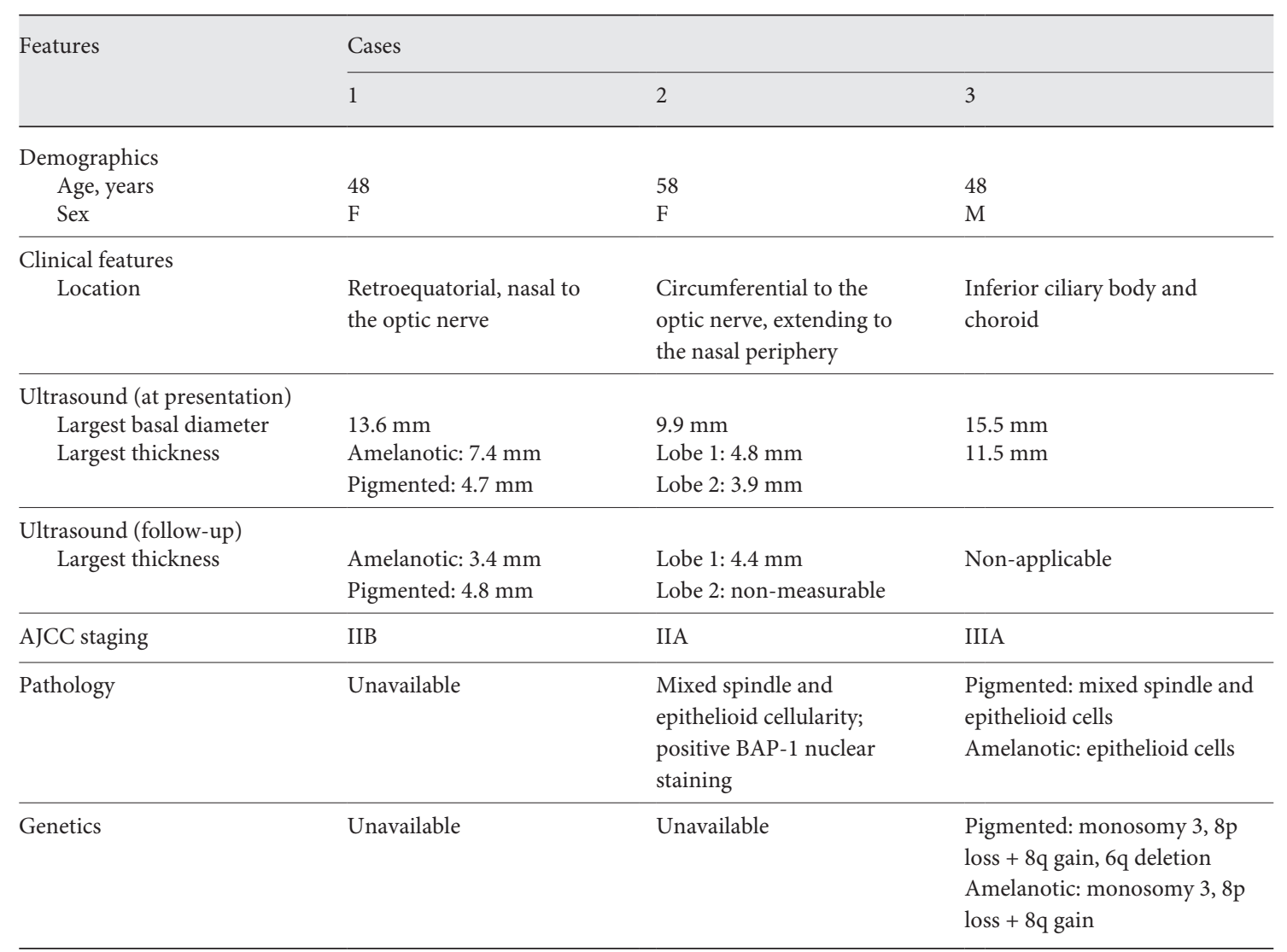

\section{Introduction}

Uveal melanoma (UM) is the most common primary intraocular tumor in adults [1]. Despite great control rates of local tumor, the 12-year mortality rate is around $40 \%$, independently of the local treatment option [2]. Several tumor features have been identified as predictors of metastatic disease, and therefore survival, and include clinical, histopathological, immunohistochemical, and genetic characteristics [1].

In contrast with most tumors, biopsies in UM patients are not only performed to confirm diagnosis, but also with the intention of obtaining a tissue specimen for prognostication and assessment of metastatic risk. The importance of an accurate prognostic information is vital for the selection of individualized screening and treatment plans. However, fine-needle aspiration biopsies have some drawbacks; apart from possible ophthalmological complications, low specimen yield and sample representativity are some of the concerns [3]. This is particularly important due to evidence of morphological and genetic heterogeneity in UM, which can lead to unpredictable tumoral behavior and compromise individualized prognostication [4].

Following a previous case report from our laboratory [4], we describe 3 cases of UM with two morphologically identifiable components, each with different behaviors and histological and genetic features (Table 1). Written consent was obtained from all patients.

\section{Case Reports}

Case 1

A 48-year-old woman presented with painless decrease of vision in her left eye (OS). On ophthalmological examination, the best corrected visual acuity (BCVA) was 20/100 OS and no remarkable anterior segment findings were apparent. On dilated fundus examination, a bilobed, elevated mass nasal to the optic nerve was present, with evidence of two morphologically distinct portions: one pigmented and the other amelanotic (Fig. 1a). Ultrasound studies confirmed the clinical diagnosis of choroidal melanoma with a bilobed configuration, with a base of $13.63 \mathrm{~mm}$ and 2 distinct lobes, the amelanotic one measuring $7.41 \mathrm{~mm}$ in
18

Ocul Oncol Pathol 2021;7:17-25 DOI: $10.1159 / 000508517$
Fonseca et al. 

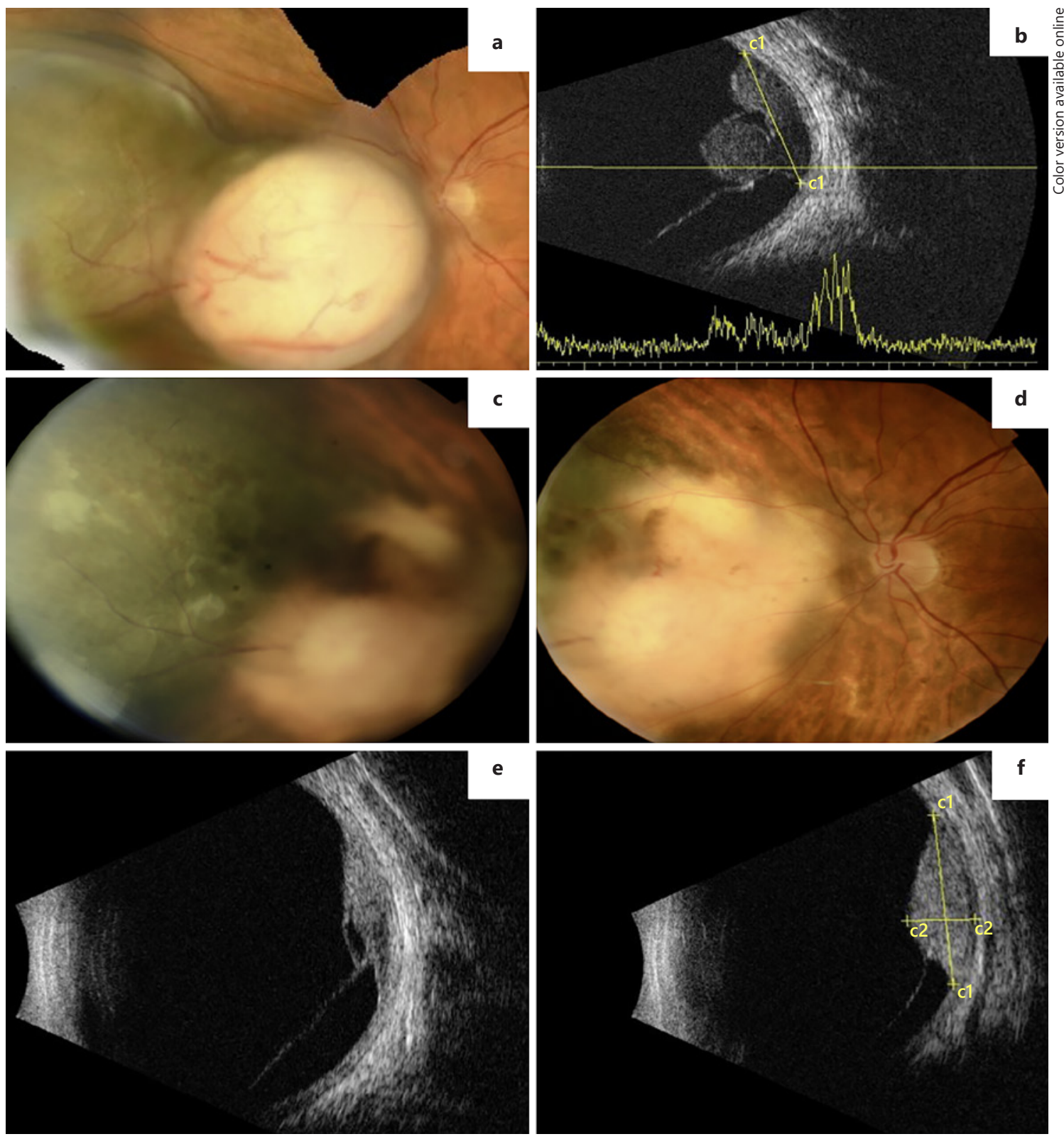

Fig. 1. Case 1. a Fundus photograph of a bilobed uveal melanoma nasal to the optic disc, composed of 2 distinct portions. b Ultrasound (US) examination showing the 2 lobes of the tumor and inferior retinal detachment. c, $\mathbf{d}$ Fundus photographs 1 year after EBT treatment clearly showing regression of the melanotic part but stability of the pigmented lobe. e, $\mathbf{f}$ US examination showing regression of the amelanotic lobe and stability of the pigmented one.

thickness and the pigmented one $4.72 \mathrm{~mm}$ (Fig. 1b). Protocol systemic workup revealed no evidence of metastatic disease and the patient underwent ${ }^{125} \mathrm{I}$ episcleral brachytherapy (EBT). During the first year of follow-up, the tumor showed regression of the amelanotic part (thickness $=3.4 \mathrm{~mm}$ ), maintaining stable dimensions in the pigmented portion $(4.8 \mathrm{~mm}$; Fig. 1c-f). The patient was diagnosed with liver metastatic disease 2 years after EBT.

A Morphologic Case Series Study

\section{Case 2}

A 58-year-old female patient presented with sudden, painless vision loss OS $(\mathrm{BCVA}=20 / 30)$. Dilated fundus examination detected the presence of a bilobed non-pigmented lesion, circumferential to the optic nerve with overlying pigment migration (Fig. 2a). The ultrasound confirmed two adjacent lobes measuring $9.3 \times 4.8$ $\mathrm{mm}$ and $9.9 \times 3.9 \mathrm{~mm}$ (Fig. $2 \mathrm{~b}$ ). The patient underwent study for choroidal metastasis with thoracic, abdominal, and pelvic com- 

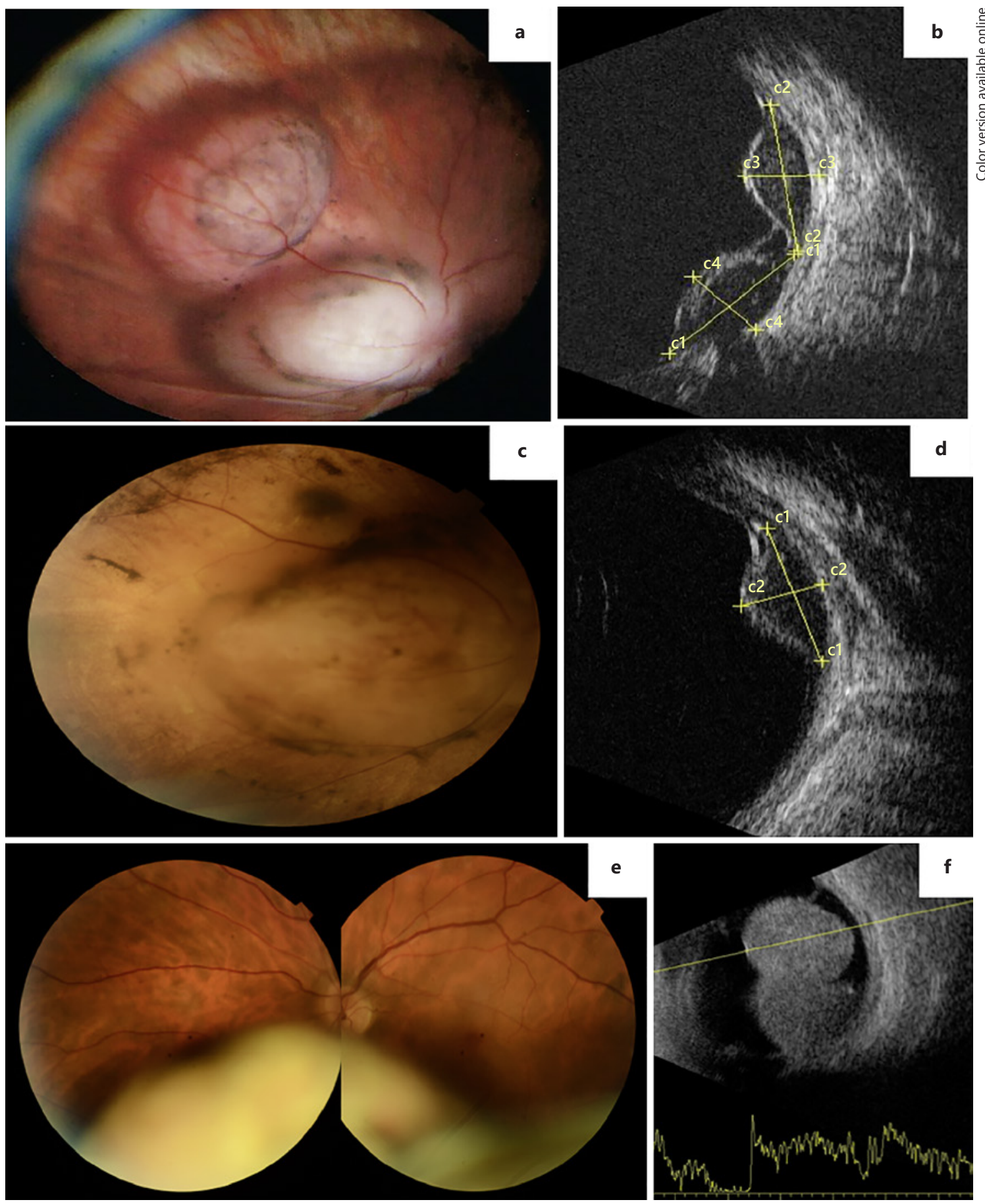

Fig. 2. Case 2. a Wide-field fundus photograph of a bilobed amelanotic uveal melanoma nasal to the optic disc. $\mathbf{b}$ US examination showing the 2 lobes of the tumor. c Fundus photograph 6 months after PBR clearly showing total regression of one of the lobes but stability of the other. $\mathbf{d}$ US examination showing only the remain- ing lobe of the tumor after PBR. Case 3. e Fundus photograph of a bilobed inferior uveal melanoma showing an amelanotic portion and a more anterior pigmented area. $f$ US examination showing a large uveal melanoma occupying a great portion of the vitreous cavity. 


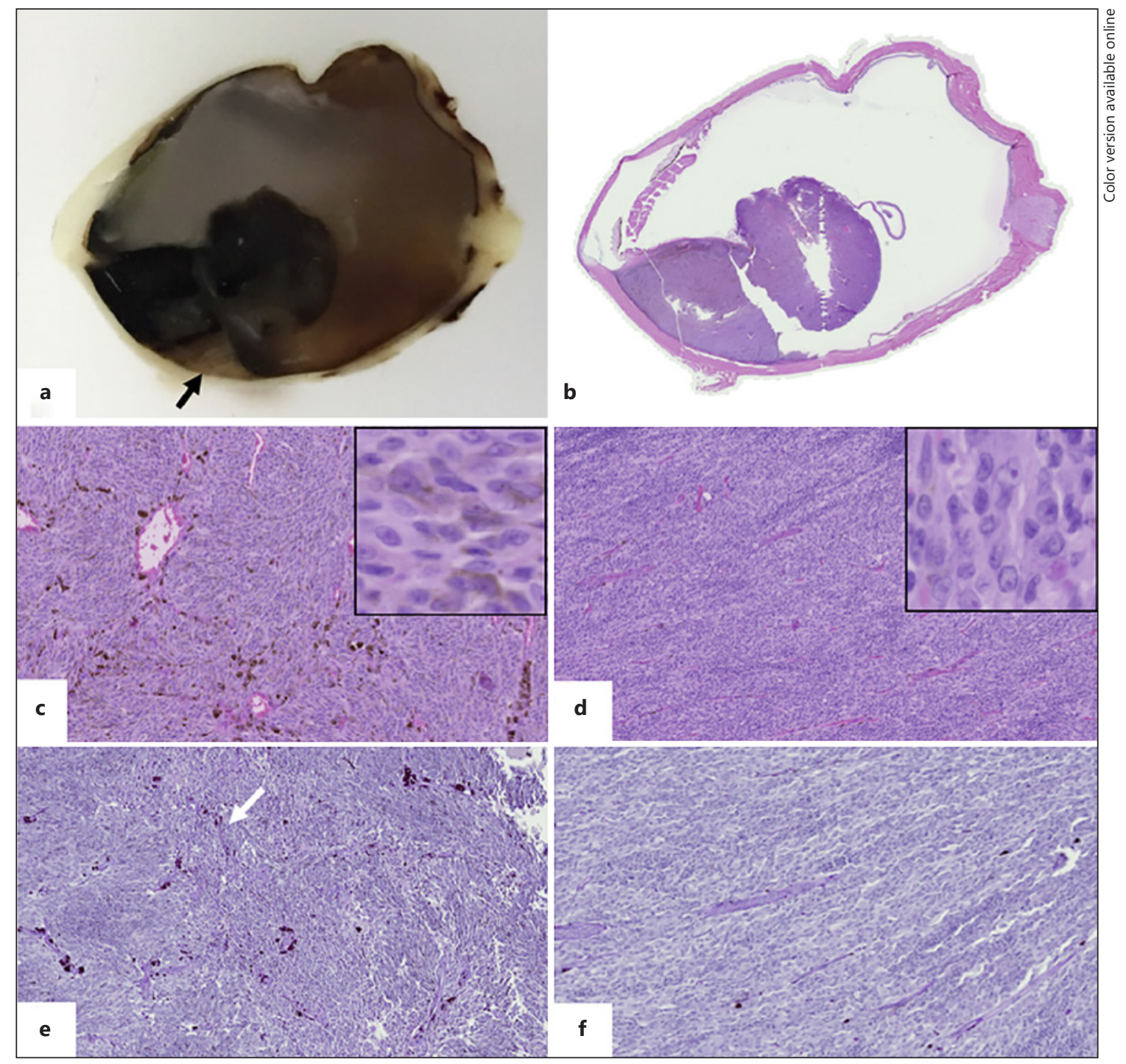

Fig. 3. Case 3. a Macroscopy of the ocular globe showing a small non-pigmented area in the base of the tumor (arrow). b Enucleated globe showing the inferior choroidal melanoma infiltrating the ciliary body (hematoxylin and eosin). c 10× magnification of the pigmented portion (inset with $40 \times$ magnification view of both epithelioid and spindle cells). d 10× magnification of the amelanotic region (inset with $40 \times$ magnification view of epithelioid cells). e PAS-stained $10 \times$ magnification view of the pigmented portion displaying vascular loops (arrow) in contrast to the same power view of the amelanotic area, without vascular loops (f). puted tomographic scans, as well as positron-emission tomography. The ancillary testing was unremarkable and no primary suspicious lesions were found. The diagnosis of UM was then considered and the patient referred for proton-beam radiotherapy (PBR). A diagnostic biopsy was performed and confirmed the diagnosis of UM with mixed spindle and epithelioid cellularity, and BAP-1 nuclear staining.

Six months after the PBR, complete regression of one of the lobes of the tumor was apparent $(8.9 \times 4.4 \mathrm{~mm})$ and radiation retinopathy developed (Fig. $2 c$, d). Systemic disease has not been detected during the current follow-up (1.5 years).
Case 3

A 48-year-old man complained of painless vision loss OS $(\mathrm{BCVA}=20 / 30)$, floaters, and photopsia for 2 months. On ophthalmological examination an inferior, bilobed, and bicolored mass was apparent (Fig. 2e). Ultrasonography was compatible with a large UM, with a basal diameter of $15.5 \mathrm{~mm}$ and thickness of $11.5 \mathrm{~mm}$ (Fig. 2f). The dimensions of the mass precluded EBT and the patient underwent left ocular globe enucleation. Systemic disease has not been detected during the current follow-up (1.5 years). 


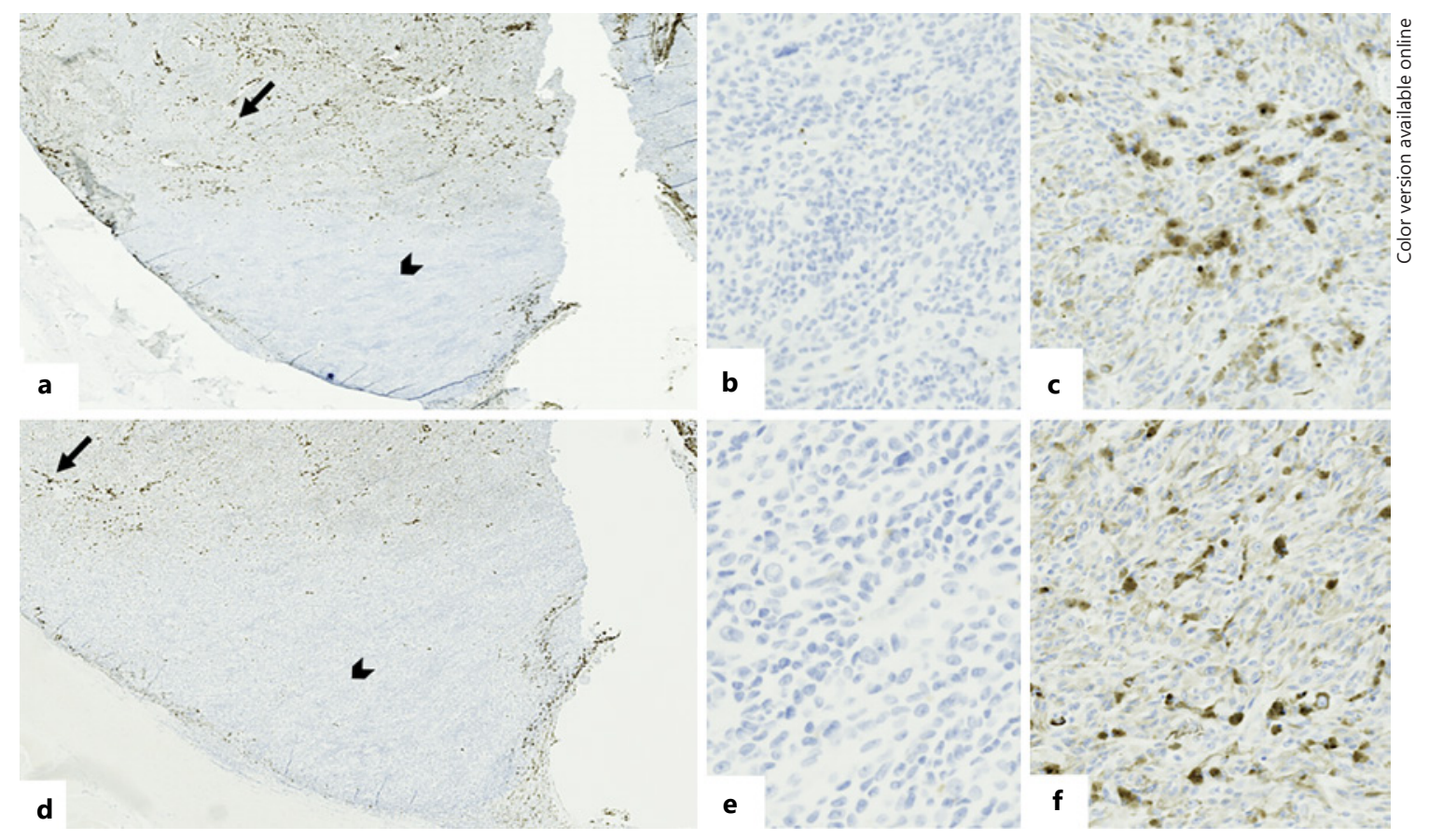

Fig. 4. Case 3. a IHC stain of the transition area between morphologically different regions of the tumor (arrow and arrowhead), showing differences in tumor lymphocytic infiltration (anti-CD3 staining). b $20 \times$ magnification of the amelanotic portion, showing absence of lymphocytic infiltration. c $20 \times$ magnification of the pigmented portion showing the presence of lymphocytes (anti-CD3 staining) infiltrating the tumor. $\mathbf{d}$ IHC stain of the transition area between morphologically different regions of the tumor (arrow and arrowhead), showing differences in macrophage infiltration (anti-CD68 staining). e 20 $\times$ magnification of the amelanotic portion, showing absence of macrophage infiltration. $\mathbf{f} 20 \times$ magnification of the pigmented portion showing the presence of macrophages (anti-CD68 staining) infiltrating the tumor.

\section{Ancillary Studies}

\section{Pathology and Immunohistochemistry}

Histopathological analysis was performed on formalin-fixed, paraffin-embedded tissues stained with hematoxylin and eosin of the enucleated specimen (case 3). Histopathological features such as vascular loops were assessed using PAS reagent. The presence of tumor-infiltrating lymphocytes and tumor-associated macrophages was evaluated using antibodies against B cells (CD20)/T cells (CD3), and anti-CD68. Cellular proliferation index was also evaluated through automated immunohistochemistry (IHC) of Ki-67, using a Ventana Benchmark (Tucson, AZ, USA).

Gross pathology evidenced an inferior choroidal and ciliary body melanoma, with a small non-pigmented part and a larger pigmented portion (Fig. 3a, b). Microscopically, these two regions showed differences, revealing a pigmented portion with a mixed cellularity, composed of both epithelioid and spindle cell patterns, and a small amelanotic region composed mainly of epithelioid cells (Fig. 3c, d). Ki-67 revealed a low mitotic index within the pigmented portion of the tumor (5\%) and little to no mitotic activity was observed in the non-pigmented area. Furthermore, the non-pigmented area revealed no vascular loops (highlighted by PAS stain in Figure 3e and f) and no lymphocytic or macrophag- ic infiltration (as highlighted by CD3/CD68 IHC), in contrast with the pigmented portion, in which vascular loops and more than 25 lymphocytes per high-power field were present (Fig. 4). The lymphocytic infiltration was more readily apparent near the base of the tumor.

\section{Genetic Studies}

DNA extraction was performed from fresh tumor samples from the pigmented and amelanotic tumoral portions of case 3 specimen, using High Pure PCR Template Preparation Kit (Roche $\mathrm{GmbH}$, Mannheim, Germany). DNA quantification and quality assessment was achieved using NanoDrop (NanoDrop Technologies, Inc., Wilmington, DE, USA). For DNA labelling and hybridization, the Agilent SurePrint G3 Human Genome 4 $\times 180 \mathrm{~K}$ platform (Agilent Technologies, Santa Clara, CA, USA) was used.

In case 3, array comparative genomic hybridization (array$\mathrm{CGH}$ ) analysis identified monosomy of the whole chromosome 3, gain of all the long arm of chromosome 8 (8q) combined with total loss of the short arm and X monosomy (loss of chromosome $\mathrm{Y}$ ) in both portions. A 108-Mb 6q deletion was only observed in the pigmented sample (Fig. 5). 


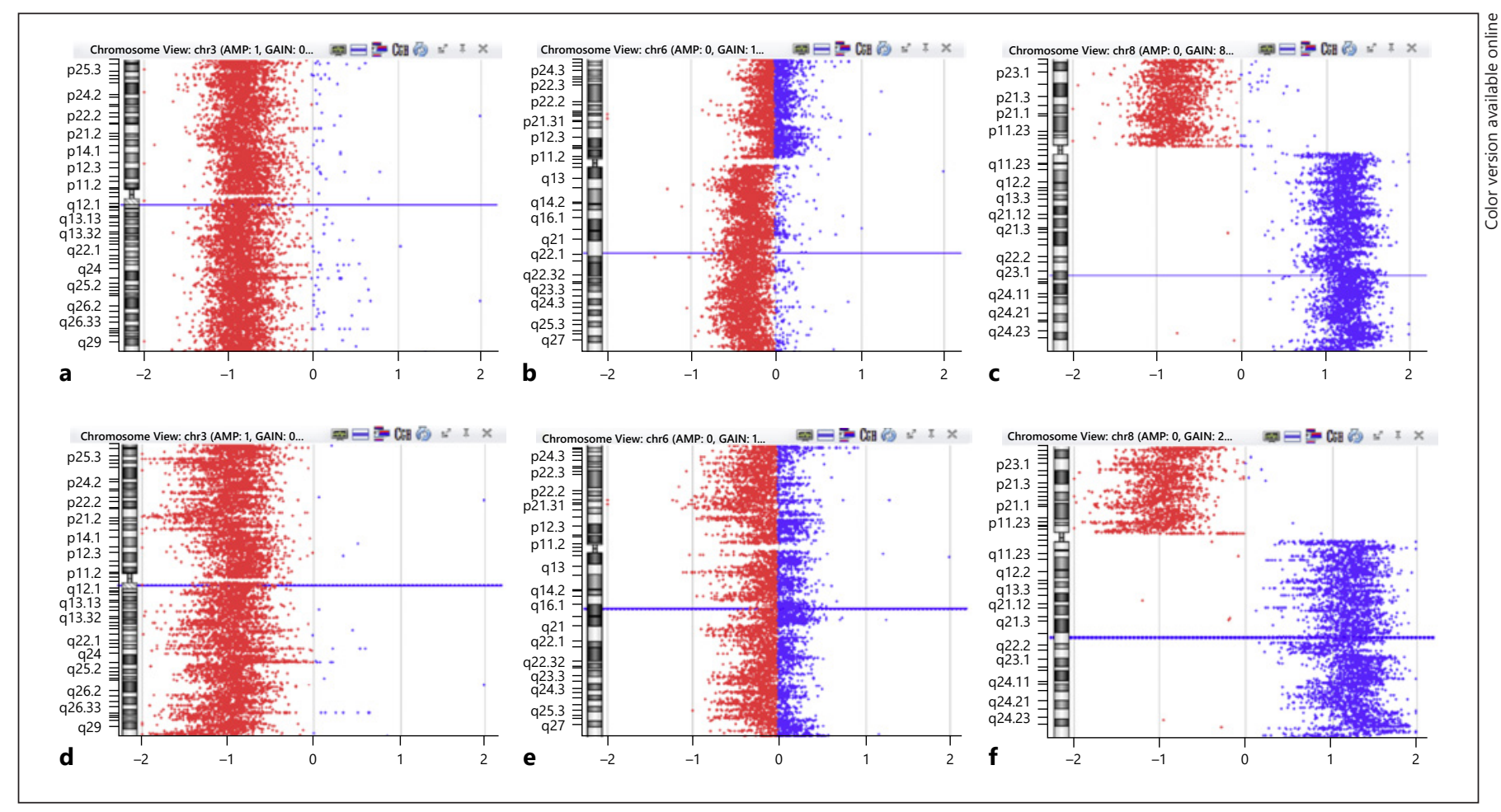

Fig. 5. a-c Chromosome view of oligoarray-CGH profile of the pigmented area. a Monosomy 3. b 6q deletion. c 8p loss combined with $8 \mathrm{q}$ gain. $\mathbf{d - f}$ Chromosome view of oligoarray-CGH profile of the amelanotic portion. $\mathbf{d}$ Monosomy 3. e $6 \mathrm{q}$ without deletion. $\mathbf{f} 8 \mathrm{p}$ loss combined with 8q gain.

\section{Discussion}

These 3 cases show examples of intratumoral heterogeneity in UM. Intratumoral heterogeneity is a common phenomenon in cancer and is related to genomic instability, leading to the formation of subclone tumor cell populations [5], particularly in medium- and large-sized tumors. Morphological heterogeneity is well documented in UM specimens of mixed cellularity, with the presence of both epithelioid and spindle cell patterns, in different proportions [5]. In UM, although risk factors include both clinical and histologic features such as epithelioid cell type, vascular mimicry, microvascular loops, and tumorinfiltrating lymphocytes and macrophages, chromosomic and genetic alterations have become increasingly important to estimate prognosis [6]. Current tests rely on either DNA or RNA extraction from tumor specimens. Techniques as fluorescence in situ hybridization (FISH), multiplex ligation-dependent probe amplification (MLPA), array-CGH, and karyotyping are commonly used for DNA analysis; gene expression profiling is the preferred technique for gene expression profiling using RNA $[3,7]$.
Cases 1 and 2 showed different clinical behaviors on different areas of the tumor, after local treatment. In case 1 , the pigmented portion of the tumor retained stable dimensions during follow-up, in contrast with the ready regression of the amelanotic part. Furthermore, the patient developed liver metastasis after 2 years and, despite the lack of histopathological and genetic studies on the tumor, we can hypothesize that at least one of the portions must have harbored high-risk features. In what concerns case 2, although morphologically similar at presentation, only one of the tumor lobes completely regressed 6 months after PBR. Histopathological examination confirmed a mixed cellular type UM with nuclear preservation of BAP-1. BAP-1 is a gene involved in various cellular processes including response to DNA damage, cell cycle regulation and cell growth. The presence of inactivating somatic or germline $B A P-1$ mutations and the loss of BAP-1 expression by lack of immunohistochemical staining have been associated with metastatic UM [6]. The patient remains currently stable with no evidence of systemic disease. 
In case 3, the enucleation specimen was obtained and screened for the classic genetic parameters of poor prognosis by array-CGH. Despite the mixed cellularity, the 2 portions displayed different main patterns, with a clear dominance of a more epithelioid-shaped pattern in the amelanotic area. Furthermore, the pigmented part also showed the presence of tumor-infiltrating lymphocytes and tumor-associated macrophages, as well as vascular loops, all of which are recognized as histopathological features of poor prognosis. More importantly, arrayCGH analysis showed typical features of poor prognosis in both portions with whole chromosome 3 loss, 8q gain, and $8 \mathrm{p}$ loss. However, when genetically comparing both portions, a $6 \mathrm{q}$ deletion was only observed in the pigmented portion, demonstrating the presence of a different subclone of tumor cells. $6 \mathrm{q}$ deletion is observed in $25-38 \%$ of $\mathrm{UM}$ cases and is also associated with poor prognosis, possibly representing a late event in tumorigenesis $[6,8]$.

Mensink et al. [5] investigated 151 UM specimens using FISH, for the presence of focal or diffuse heterogeneity of monosomy 3 . These authors concluded that despite finding subclones with different percentages of chromosome 3 monosomy in 10 cases, none of those patients required adjustment of the risk calculation for metastatic disease and it did not affect survival rates [5]. Also, Bagger et al. [9] compared transvitreal retinochoroidal biopsies of 27 UM to their total genetic profile and found that tumors were heterogenic regarding chromosome 8 , whereas chromosome 3 aberrations were often present in most of the tumor, rendering biopsy valid for differentiating high- and low-risk patients. Similarly, Dopierala et al. [10] compared MLPA data from different areas in $32 \mathrm{UM}$ and showed loci heterogeneity in $75 \%$ of the tumors, most commonly affecting 3p12.2, 6p21.2, and 8q11.23 loci.

In contrast, a previous case published by our laboratory showed a tumor with rapidly enlarging pigmented portion with an apparent transformation from an initial amelanotic indolent growing mass [4]. In the non-pigmented portion, normal chromosomal 3 heterodisomy was apparent, in opposition to the pigmented area, with evident chromosomal 3 monosomy allied to histopathological features of poor prognosis. This highlights that caution should be paramount when determining prognosis based on fine-needle aspiration biopsy, especially because tumor samples are obtained by a single pass. A similar case was also reported by White et al. [11] demonstrating distinct pigmented and non-pigmented areas in a UM patient, with differences in histopathological and cytogenetic features between the 2 portions. These authors also showed that duplication of chromosome 3 had occurred, concluding that isodisomy 3 developed during clonal evolution of the non-pigmented portion, from the pigmented one.

To conclude, we present a case series with three examples of UM intratumoral heterogeneity in medium and large UM. Although prognostication in UM has achieved high precision, rendering tumor biopsies a standard approach, some limitations cannot be ignored, the most relevant being spatial and temporal tumor heterogeneity. The clinical recognition of heterogeneous shape, color, and size of the tumor at presentation should guide the clinician to consider the possibility of genomic heterogeneity and the need to sample different areas of the tumor during prognostic fine-needle aspiration biopsies. Furthermore, an uneven response to local treatment is also a possibility to be considered.

\section{Statement of Ethics}

Written consent was obtained from all patients included in this study, for publication of their cases and images. This study was conducted ethically in accordance with the World Medical Association Declaration of Helsinki and approved by the Institute's Ethics Committee.

\section{Conflict of Interest Statement}

The authors have no conflict of interest to declare.

\section{Funding Sources}

No funding was obtained for the purpose of this study from any of the authors.

\section{Author Contributions}

C.F.: conception, data acquisition, data interpretation, writing (original draft), project administration; R.P.-P.: conception, writing (review and editing); S.B.: data acquisition, data interpretation, writing (review and editing); L.M.P.: data acquisition, data interpretation, formal analysis, writing (review and editing); J.F.: data acquisition, data interpretation. I.M.C.: data interpretation, writing (review and editing); M.N.B.: conception, data interpretation, writing (review and editing); R.P.: conception, data acquisition, data interpretation, writing (review and editing), supervision.
Fonseca et al. 


\section{References}

1 Kaliki S, Shields CL. Uveal melanoma: relatively rare but deadly cancer. Eye (Lond). 2017 Feb; 31(2): 241-57.

2 Collaborative Ocular Melanoma Study Group. The COMS randomized trial of iodine 125 brachytherapy for choroidal melanoma: $\mathrm{V}$. Twelve-year mortality rates and prognostic factors: COMS report No. 28. Arch Ophthalmol. 2006 Dec;124(12):1684-93.

3 Frizziero L, Midena E, Trainiti S, Londei D, Bonaldi L, Bini S, et al. Uveal Melanoma Biopsy: A Review. Cancers (Basel). 2019 Jul; 11(8): 1075 .

4 Lim LA, Miyamoto C, Blanco P, Bakalian S, Burnier MN Jr. Case report: an atypical peripapillary uveal melanoma. BMC Ophthalmol. $2014 \mathrm{Feb} ; 14(1): 13$.
5 Mensink HW, Vaarwater J, Kiliç E, Naus NC, Mooy N, Luyten G, et al. Chromosome 3 intratumor heterogeneity in uveal melanoma. Invest Ophthalmol Vis Sci. 2009 Feb;50(2): 500-4.

6 Kaliki S, Shields CL, Shields JA. Uveal melanoma: estimating prognosis. Indian J Ophthalmol. 2015 Feb;63(2):93-102.

7 Onken MD, Worley LA, Char DH, Augsburger JJ, Correa ZM, Nudleman E, et al. Collaborative Ocular Oncology Group report number 1: prospective validation of a multi-gene prognostic assay in uveal melanoma. Ophthalmology. 2012 Aug;119(8):1596-603.

8 van den Bosch T, Kilic E, Paridaens D, de Klein A. Genetics of uveal melanoma and cutaneous melanoma: two of a kind? Dermatol Res Pract. 2010;2010:360136.
9 Bagger M, Andersen MT, Heegaard S, Andersen MK, Kiilgaard JF. Transvitreal Retinochoroidal Biopsy Provides a Representative Sample From Choroidal Melanoma for Detection of Chromosome 3 Aberrations. Invest Ophthalmol Vis Sci. 2015 Sep;56(10):5917-24.

10 Dopierala J, Damato BE, Lake SL, Taktak AF, Coupland SE. Genetic heterogeneity in uveal melanoma assessed by multiplex ligation-dependent probe amplification. Invest Ophthalmol Vis Sci. 2010 Oct;51(10):4898-905.

11 White VA, McNeil BK, Thiberville L, Horsman DE. Acquired homozygosity (isodisomy) of chromosome 3 during clonal evolution of a uveal melanoma: association with morphologic heterogeneity. Genes Chromosomes Cancer. 1996 Feb;15(2):138-43. 